\title{
Simulation-guided engineering of antibiotics for improved bacterial uptake
}

\author{
Ricardo J. Ferreira, ${ }^{1}$ Valeria Aguilar ${ }^{1}$, Ana M. Villamil Giraldo ${ }^{1}$, Peter M. Kasson ${ }^{1,2^{*}}$ \\ ${ }^{1}$ Science for Life Laboratory, Department of Cell and Molecular Biology, Uppsala University, \\ BMC Box 596, S-751 23 Uppsala, Sweden. \\ ${ }^{2}$ Departments of Biomedical Engineering and Molecular Physiology and Biological Physics, \\ University of Virginia, Charlottesville, VA 22908, USA. \\ * kasson@virginia.edu
}

\begin{abstract}
The Gram-negative bacterial outer membrane poses a major obstacle to the development of much-needed antibiotics against drug-resistant infections. Its chemical composition and porin proteins differ from Gram-positive bacteria and mammalian cells, and heuristics developed for mammalian cell uptake apply poorly. Recently, machinelearning methods have predicted small-molecule uptake into Gram-negative bacteria, offering the possibility to rationally optimize this aspect of antibiotic lead development. Here, we report physics-based methods to prospectively predict Gram-negative bacterial uptake, select, and synthesize promising chemical derivatives targeting $E$. coli DNA gyrase B. Our methods do not require empirical parameterization and are readily adaptable to new chemical scaffolds. These physics-based predictions well capture experimentally measured uptake $(r>0.95)$ and are indeed predictive of antimicrobial activity $(r>0.92)$. These methods can be used prospectively in combination with targetbinding simulations to optimize both bacterial uptake and target binding, overcoming important barriers to antibiotic lead generation before small-molecule synthesis.
\end{abstract}

\section{Introduction}

Antibiotic-resistant bacterial infections are an increasing global health threat and are estimated to surpass cancer as a cause of death by $2050^{1}$. Responding to this challenge requires improved diagnostics, improved antimicrobial stewardship, and critically the development of new antibiotics ${ }^{2,3,4}$. New classes of antibiotics active against Gramnegative bacteria have been particularly difficult to develop because in order to reach their targets antibiotics must traverse the Gram-negative outer membrane, which poses a permeability barrier 5, 6,7 Depending on the antibiotic chemistry, inner membrane permeability and efflux can also be substantial contributors, but here we concentrate on the outer membrane, which is a major determining factor for several major classes of antibiotics. This outer membrane differs from both mammalian cells and gram-positive bacterial membranes in the high proportion of lipopolysaccharide (LPS) in its outer leaflet and the semi-specific uptake porins, creating very different criteria for efficient antibiotic uptake $^{8,9,10}$. In fact, most FDA-approved antibiotics against Gram-negative bacteria 
violate at least one of the heuristic rules developed to predict mammalian cell uptake ${ }^{5}$. Approaches for selecting and optimizing lead compounds largely rely upon empirical measurements of bacterial growth inhibition, and it has been much easier to rationally or computationally optimize on-target activity than cellular accumulation.

Computational approaches to optimize compound accumulation in Gramnegative bacteria have used a combination of machine learning on molecular features and physics-based methods. Gram-negative accumulation results from a combination of uptake, efflux, and intracellular metabolism. For many hydrophilic compounds, uptake is a good predictor of intracellular accumulation ${ }^{11,12,13}$. Hergenrother and colleagues have reported good success with machine learning based on accumulation data ${ }^{14,15}$. An alternate approach has been to parameterize molecular descriptors based on physics-based simulations ${ }^{16,17,18,19,20,21}$ or to forgo computational optimization and focus solely on medium-scale screening approaches ${ }^{22,23,24}$. We have previously shown how a simulation-based approach can prospectively predict antibiotic uptake without explicit parameterization ${ }^{12}$. Here, we test the ability of such an approach to guide compound selection for increased uptake and increased antimicrobial potency prior to chemical synthesis.

DNA gyrase $B$ has been an attractive yet challenging target for antibacterial drug development $25,26,27,28,29,30$. The most successful inhibitor of this target, novobiocin, was withdrawn due to lack of safety or effectiveness ${ }^{31}$. The ATP-binding pocket of DNA gyrase B has been the subject of renewed preclinical interest, but Gram-negative uptake has been a persistent challenge ${ }^{30}$. This is evidenced by poor minimum inhibitory concentrations (MICs) even when biochemical inhibitory concentrations $\left(\mathrm{IC}_{50} \mathrm{~s}\right)$ against $E$. coli DNA gyrase B are reported in the nanomolar range. As a proof of concept, we selected flavanone compounds for optimization. Flavonoids have been the subject of previous medicinal chemistry efforts against $E$. coli DNA gyrase $B^{32,33,34}$, and the flavanone naringenin has been a particularly promising starting point, as it provides a readily derivatized scaffold.

In this paper we aim to optimize the bacterial permeabilities for a small set of naringenin hydrazone derivatives while maintaining or improving binding affinity to $E$. coli DNA gyrase B. Prior to synthesizing these derivatives, we estimated both the relative free-energies of binding and the outer-membrane permeability, using calculated OmpF permeability as a surrogate for full E. coli outer membrane permeability as we have previously demonstrated ${ }^{12}$. Improved outer-membrane permeability and equal or better binding affinity were used as selection criteria for synthesizing derivatives and testing them experimentally. The resulting experimental data on both outer-membrane uptake and cellular growth inhibition correlate well with our predictions, demonstrating that molecular simulations of membrane permeability can be used in a prospective manner to guide selection and chemical synthesis of potential antibacterial lead compounds. 

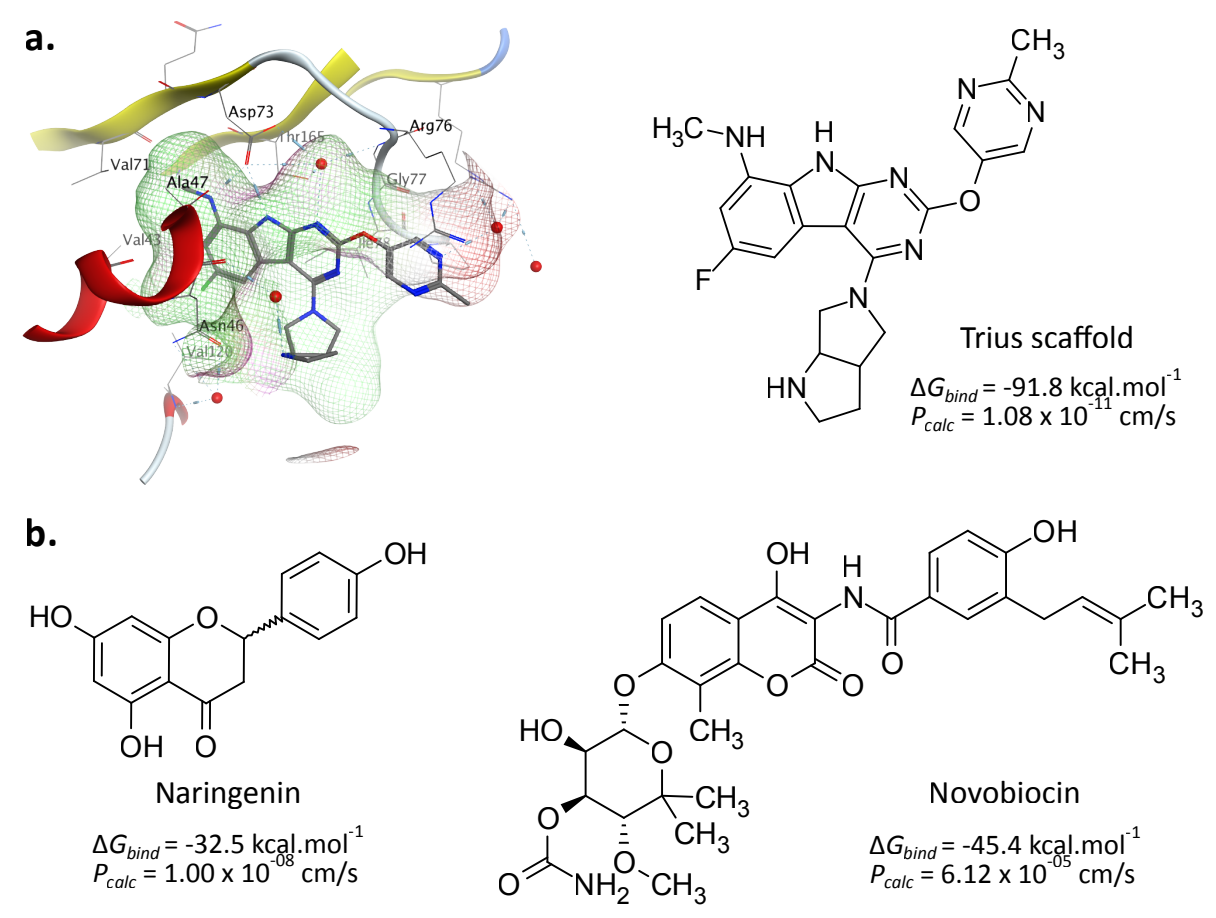

Figure 1. DNA gyrase B binding pocket and inhibitors. Rendered in panel (a) is the ATPbinding pocket of $E$. coli DNA gyrase $B$ with an inhibitor bound, based on co-crystal structure $4 \mathrm{KFG}$. The chemical structure of the co-crystalized ligand is shown with approximate binding free energy (MM-PBSA) and predicted outer-membrane permeability. Panel (b) similarly displays the naringenin starting scaffold and the related antibiotic novobiocin with their predicted binding and permeability values.

\section{Results}

To test the hypothesis that outer-membrane permeability simulations can guide antibiotic lead optimization, we selected a set of synthetically accessible naringenin derivatives for simulation and assessment. The naringenin scaffold can bind and inhibit the ATP-binding pocket of $E$. coli DNA gyrase B with moderate affinity and, we predict, poor-to-moderate permeability, leading to a suboptimal MIC in bacteria $(428 \mu \mathrm{g} / \mathrm{mL})$. Naringenin is commercially available and readily derivatized, providing a good proof-ofconcept system for optimization of antibiotic leads based on prospective prediction of bacterial uptake. Hydrazone derivatives were selected based on examination of the binding pose of naringenin to DNA gyrase $B$ and the potential to reinforce hydrogen bonding with Asn46 and Asp73 (see Supplement for further discussion).

We therefore predicted the $E$. coli outer-membrane permeability (here estimated as permeability via OmpF, see prior studies ${ }^{12,16}$ for discussion of this approximation) and the approximate target binding affinity of a set of 29 derivatives, as described below. Predicted permeability and approximate target binding are listed in Table S2. 61 derivatives (hydrazone-linked flavanones, see Methods) were initially evaluated by molecular docking. Of these, 29 were predicted to maintain a binding mode similar to that of a previously co-crystallized inhibitor (Figure 1A; Tables S1-S2 and Figures S1S2). Approximate receptor-binding energies and membrane permeabilities were 
predicted for each of these. Six of these compounds (21\%) were predicted to have lower permeabilities than naringenin (Figure 1B). Herein, compounds D19 (carboxylate derivative) D29 (quinoxaline), D5 (m-ethyl benzoate), D13 (o-nitrophenyl), D15 (indan), and D21 (imidazole) were predicted to have permeabilities lower than $1 \times 10^{-8} \mathrm{~cm} / \mathrm{s}$. Approximate binding energies calculated via MM-PBSA are listed in the Supplement. These compounds were then filtered based on 1) prediction of improved bacterial permeability, 2) prediction of maintained or improved target binding, and 3) commercial availability of starting materials to yield the compound set shown in Figure 2. Compound $\mathbf{1}$ is the starting compound; compounds $\mathbf{2 - 4}$ are previously synthesized compounds ${ }^{35}$ that were also predicted to have binding energies and permeabilities within the filter criteria; compounds 5-9 were synthesized based on the criteria above. Compound 10 was synthesized as a negative control based on its poor predicted permeability.

a.<smiles>C/C=C(\C)[C@@H]1CC(=O)c2c(O)cc(O)cc2O1</smiles>

2 $\Delta G_{\text {bind }}=-59.6 \mathrm{kcal} . \mathrm{mol}^{-1}$
$P_{\text {calc }}=1.30 \times 10^{-03} \mathrm{~cm} / \mathrm{s}$<smiles>Cc1cccc([N+](=O)[O-])c1</smiles>

5 $\Delta G_{\text {bind }}=-65.4 \mathrm{kcal} . \mathrm{mol}^{-1}$ $P_{\text {calc }}=7.80 \times 10^{-08} \mathrm{~cm} / \mathrm{s}$<smiles>Cc1cc(C)c2ccccc2n1</smiles>

8 $\Delta G_{\text {bind }}=-61.2 \mathrm{kcal} . \mathrm{mol}^{-1}$
$P_{\text {calc }}=7.21 \times 10^{-06} \mathrm{~cm} / \mathrm{s}$<smiles>Cc1ccccc1</smiles>

3 $\Delta G_{\text {bind }}=-59.0 \mathrm{kcal} . \mathrm{mol}^{-1}$ $P_{\text {calc }}=4.37 \times 10^{-03} \mathrm{~cm} / \mathrm{s}$<smiles>Cc1cccc(Br)c1</smiles>
6 $\Delta G_{\text {bind }}=-63.9 \mathrm{kcal} . \mathrm{mol}^{-1}$ $P_{\text {calc }}=2.28 \times 10^{-03} \mathrm{~cm} / \mathrm{s}$<smiles>Cc1ccc(Cl)nn1</smiles>

9

$\Delta G_{\text {bind }}=-59.6 \mathrm{kcal} \cdot \mathrm{mol}^{-1}$ $P_{\text {calc }}=6.42 \times 10^{-05} \mathrm{~cm} / \mathrm{s}$<smiles>[R]NN=C1C[C@@H](c2ccc(O)cc2)Oc2cc(O)cc(O)c21</smiles><smiles>Cc1cnccn1</smiles>

4

$\Delta G_{\text {bind }}=-60.8 \mathrm{kcal} \cdot \mathrm{mol}^{-1}$ $P_{\text {calc }}=1.36 \times 10^{-07} \mathrm{~cm} / \mathrm{s}$<smiles>Clc1ccc(CI)cc1Cl</smiles>

7

$\Delta G_{\text {bind }}=-59.5 \mathrm{kcal} . \mathrm{mol}^{-1}$ $P_{\text {calc }}=5.86 \times 10^{-06} \mathrm{~cm} / \mathrm{s}$<smiles>CCOC(=O)c1cccc(C)c1</smiles>
10 $\Delta G_{\text {bind }}=-68.8 \mathrm{kcal} \cdot \mathrm{mol}^{-1}$ $P_{\text {calc }}=4.31 \times 10^{-12} \mathrm{~cm} / \mathrm{s}$

Figure 2. Naringenin derivatives tested. Schematized in panel (a) is the derivatization strategy used to prepare derivatives of naringenin (1). Reaction conditions were hydrazine (R-NH-NH (2.0 eq.) in ethanol with a catalytic amount of acetic acid, in a reflux apparatus for 12-48 $\mathrm{h}$. Schematized in panel (b) are the naringenin derivatives predicted and tested, with their predicted approximate binding free energies and permeabilities. All biological activities and physicochemical properties can be found in Tables 1 and S3). 
Selected compounds were synthesized and tested experimentally for bacterial outer membrane uptake, inhibition of DNA gyrase, and inhibition of bacterial growth. The compound set also included three previously synthesized flavanone derivatives and three comparator compounds predicted to have low bacterial membrane permeability. Synthesis and characterization is described in the Methods. Bacterial outer membrane uptake was measured using an assay for osmotic swelling of bacterial outer membrane vesicles (OMV) that we have reported previously ${ }^{12}$. Results of this assay show a strong correlation (Pearson $r$ 0.95) of outer-membrane uptake of the synthesized derivatives with predicted permeabilities over an 8-log range (Fig. 3). Notably, compounds $2\left(P_{\text {calc }} 1.30 \times 10^{-3} \mathrm{~cm} / \mathrm{s} ; P_{\text {exp }} 99.6 \%\right), 3\left(P_{\text {calc }} 4.37 \times 10^{-3} \mathrm{~cm} / \mathrm{s} ; P_{\text {exp }} 103.8 \%\right)$ and $6\left(P_{\text {calc }} 2.28 \times 10^{-3} \mathrm{~cm} / \mathrm{s} ; P_{\text {exp }} 85.2 \%\right)$ had permeabilities comparable to that of glycine $\left(P_{\text {calc }} 5.32 \times 10^{-2} \mathrm{~cm} / \mathrm{s} ; P_{\text {exp }} 100 \%\right)$. Our prior validation studies on simulated uptake via OmpF and OMV swelling show similarly good correlations between simulations and accumulation measured via mass spectrometry ${ }^{12}$ and also present validation data on OmpF-deletion strains. The logarithmic relationship between permeability coefficients and osmotic swelling is well described ${ }^{36}$, and the final concentration of antibiotic scales with OMV swelling.

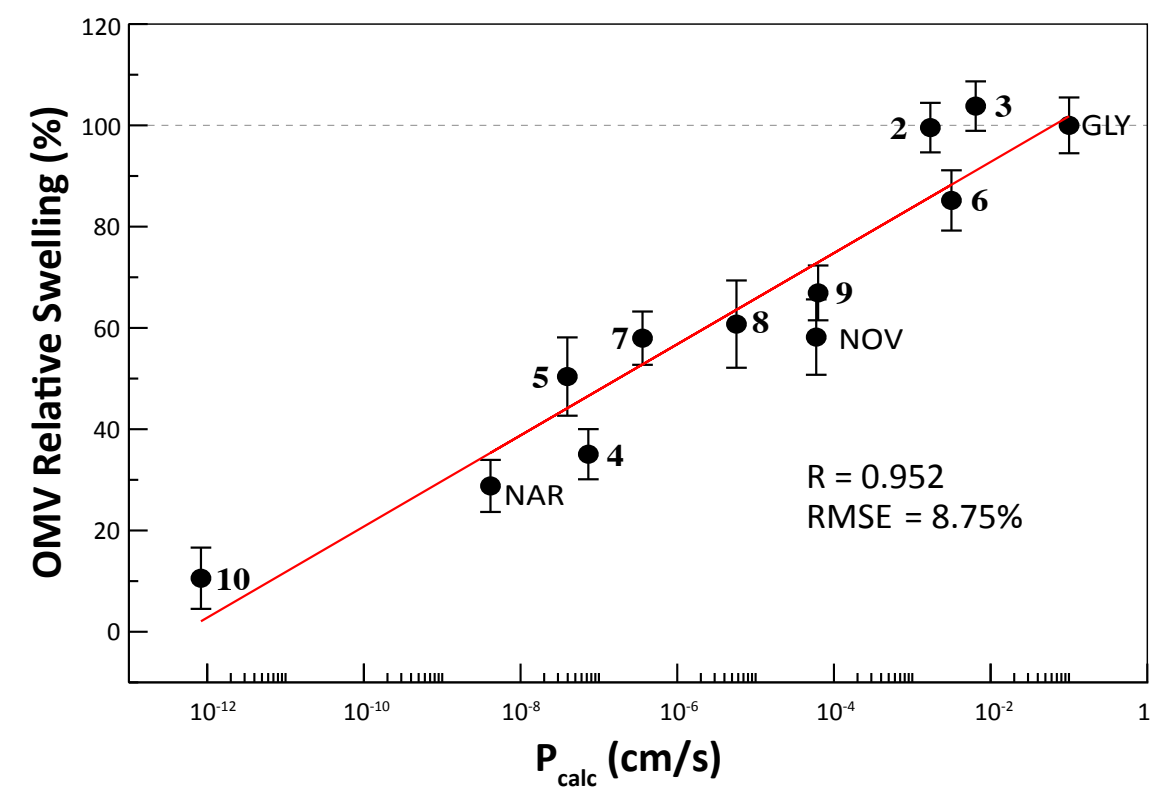

Figure 3. Predicted permeabilities correlate well with measured outer-membrane swelling. Plotted are semilogarithmic correlations of experimental OMV swelling rates and predicted permeabilities in $E$. coli outer membrane vesicles containing OmpF for naringenin (NAR), glycine (GLY), novobiocin (NOV) and the flavanone derivatives 2-10. Permeabilities are normalized with respect to glycine.

We next tested the antibacterial activity of each compound against the $E$. coli strain MG1655. Minimum inhibitory concentrations (MIC) were determined via microbroth dilution and $\mathrm{EC}_{50}$ values were plotted against predicted outer-membrane permeability values in Fig. 4. Again, $\mathrm{EC}_{50}$ values show a strong correlation with the logarithm of predicted permeability (Pearson $r$ 0.93), with $86 \%$ of variance in MIC 
explained by predicted permeability. Compounds 6, 7 and 10 also displayed a moderate bacteriostatic effect at lower concentrations, retaining 20 to $40 \%$ growth inhibition as low as $1.0 \mu \mathrm{g} / \mathrm{mL}$. $\mathrm{EC}_{50}$ for ciprofloxacin and novobiocin were measured as controls at 4 $\mathrm{ng} / \mathrm{mL}$ and $7.9 \mu \mathrm{g} / \mathrm{mL}$, respectively. The strong correlation suggests that membrane permeability is a first-order determinant of antimicrobial activity for the compounds tested, in accordance with previous predictions ${ }^{12}$. These data take the additional step to demonstrate that permeability can be optimized prospectively based on simulations to improve antimicrobial activity.

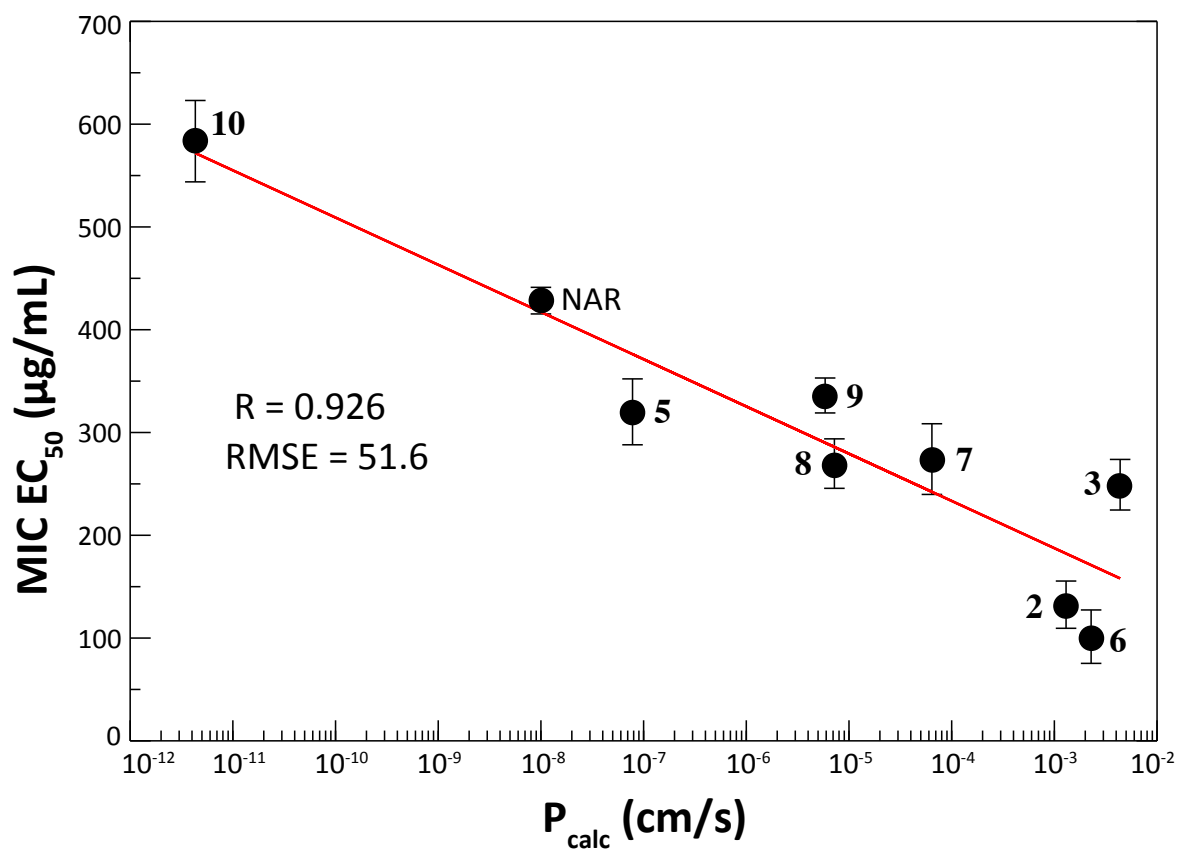

Figure 4. Predicted outer-membrane permeabilities correlate well with antimicrobial activity. Plotted are semilogarithmic correlations of the growth inhibitory concentrations (MIC $\left.\mathrm{EC}_{50}\right)$ with calculated OmpF outer-membrane permeabilities $\left(P_{\text {calc }}\right)$ for naringenin (NAR) and all flavanone derivatives except 4 . Compound $4 \mathrm{EC}_{50}$ was determined to be $>400 \mu \mathrm{g} / \mathrm{mL}$.

Table 1. $E_{50}$ values for growth inhibition ( $\left.M I C E_{50}\right)$, DNA gyrase $B$ supercoiling inhibitory activities $\left(G y r \mid C_{50}\right)$, relative free-energies of binding $\left(\Delta G_{\text {bind }}\right)$, predicted $\left(P_{\text {calc }}\right)$ and experimental $\left(P_{\text {exp }}\right)$ OmpF permeabilities for compounds 1-10.

\begin{tabular}{|c|c|c|c|c|c|c|c|}
\hline & $\begin{array}{c}\text { MIC EC }_{50} \\
(\mu \mathrm{g} / \mathrm{mL})\end{array}$ & $\begin{array}{c}\text { MIC EC }_{50} \\
(\mu \mathrm{M})\end{array}$ & $\begin{array}{l}\text { Gyr IC } 50 \\
(\mu \mathrm{g} / \mathrm{mL})\end{array}$ & $\begin{array}{c}\text { Gyr IC }{ }_{50} \\
(\mu \mathrm{M})\end{array}$ & $\begin{array}{c}\Delta G_{\text {bind }} \\
\text { (kcal/m } \\
\text { ol) }\end{array}$ & $\begin{array}{c}P_{\text {calc }} \\
(\mathrm{cm} / \mathrm{s})\end{array}$ & $\begin{array}{l}P_{\text {exp }} \\
(\%)\end{array}$ \\
\hline 1 & $428 \pm 13$ & $1572 \pm 22$ & $299 \pm 8$ & $1098 \pm 30$ & $-32 \pm 5$ & $1.0 \mathrm{E}-08 \pm 9.8 \mathrm{E}-10$ & $29 \pm 5$ \\
\hline 2 & $132 \pm 23$ & $300 \pm 52$ & $28 \pm 7$ & $64 \pm 17$ & $-60 \pm 4$ & $1.3 \mathrm{E}-03 \pm 1.2 \mathrm{E}-04$ & $99 \pm 5$ \\
\hline 3 & $249 \pm 25$ & $688 \pm 68$ & $17 \pm 3$ & $46 \pm 9$ & $-59 \pm 4$ & $4.4 \mathrm{E}-03 \pm 5.2 \mathrm{E}-04$ & $104 \pm 5$ \\
\hline 4 & n.d. & n.d. & n.d. & n.d. & $-61 \pm 5$ & $1.4 \mathrm{E}-07 \pm 9.1 \mathrm{E}-09$ & $35 \pm 5$ \\
\hline 5 & $320 \pm 32$ & $786 \pm 79$ & $33 \pm 6$ & $81 \pm 14$ & $-65 \pm 4$ & $7.8 \mathrm{E}-08 \pm 5.8 \mathrm{E}-09$ & $50 \pm 8$ \\
\hline 6 & $101 \pm 26$ & $230 \pm 59$ & $29 \pm 5$ & $65 \pm 12$ & $-64 \pm 4$ & $2.3 \mathrm{E}-03 \pm 3.0 \mathrm{E}-04$ & $85 \pm 6$ \\
\hline 7 & $336 \pm 17$ & $756 \pm 38$ & $72 \pm 7$ & $161 \pm 15$ & $-60 \pm 5$ & $5.9 \mathrm{E}-06 \pm 5.7 \mathrm{E}-07$ & $58 \pm 5$ \\
\hline 8 & $270 \pm 24$ & $631 \pm 56$ & $163 \pm 7$ & $382 \pm 17$ & $-61 \pm 6$ & $7.2 \mathrm{E}-06 \pm 7.2 \mathrm{E}-07$ & $61 \pm 9$ \\
\hline
\end{tabular}




\begin{tabular}{|l|c|c|c|c|c|c|c|}
\hline $\mathbf{9}$ & $294 \pm 19$ & $737 \pm 48$ & $91 \pm 6$ & $228 \pm 14$ & $-60 \pm 4$ & $6.4 \mathrm{E}-05 \pm 5.5 \mathrm{E}-06$ & $67 \pm 5$ \\
\hline $\mathbf{1 0}$ & $583 \pm 40$ & $1342 \pm 109$ & $18 \pm 5$ & $42 \pm 12$ & $-69 \pm 5$ & $4.3 \mathrm{E}-12 \pm 2.9 \mathrm{E}-13$ & $11 \pm 6$ \\
\hline
\end{tabular}

To rule out the effect of changes to DNA gyrase inhibition, we tested the ability of each compound to inhibit $E$. coli DNA gyrase B in a cell-free context using a DNA supercoiling assay with purified gyrase enzyme. As shown in Fig. 5, measured $\mathrm{IC}_{50}$ values for DNA gyrase B inhibition correlate moderately with crude estimates of target binding affinity from MM-PBSA calculations (Pearson $r=0.87$ however Spearman rho 0.27 ) but minimally with microbiological $\mathrm{EC}_{50}$ values (Pearson $r=0.26$ ), suggesting that the improvement in antimicrobial activity was indeed due to enhanced bacterial uptake rather than enhanced biochemical activity once inside the cell. Given the rough approximation involved in MM-PBSA calculations (there exist more accurate methods for estimating experimental binding free energies), these data provide supporting evidence that permeability can be optimized independently of target-binding affinity to improve antimicrobial activity. 

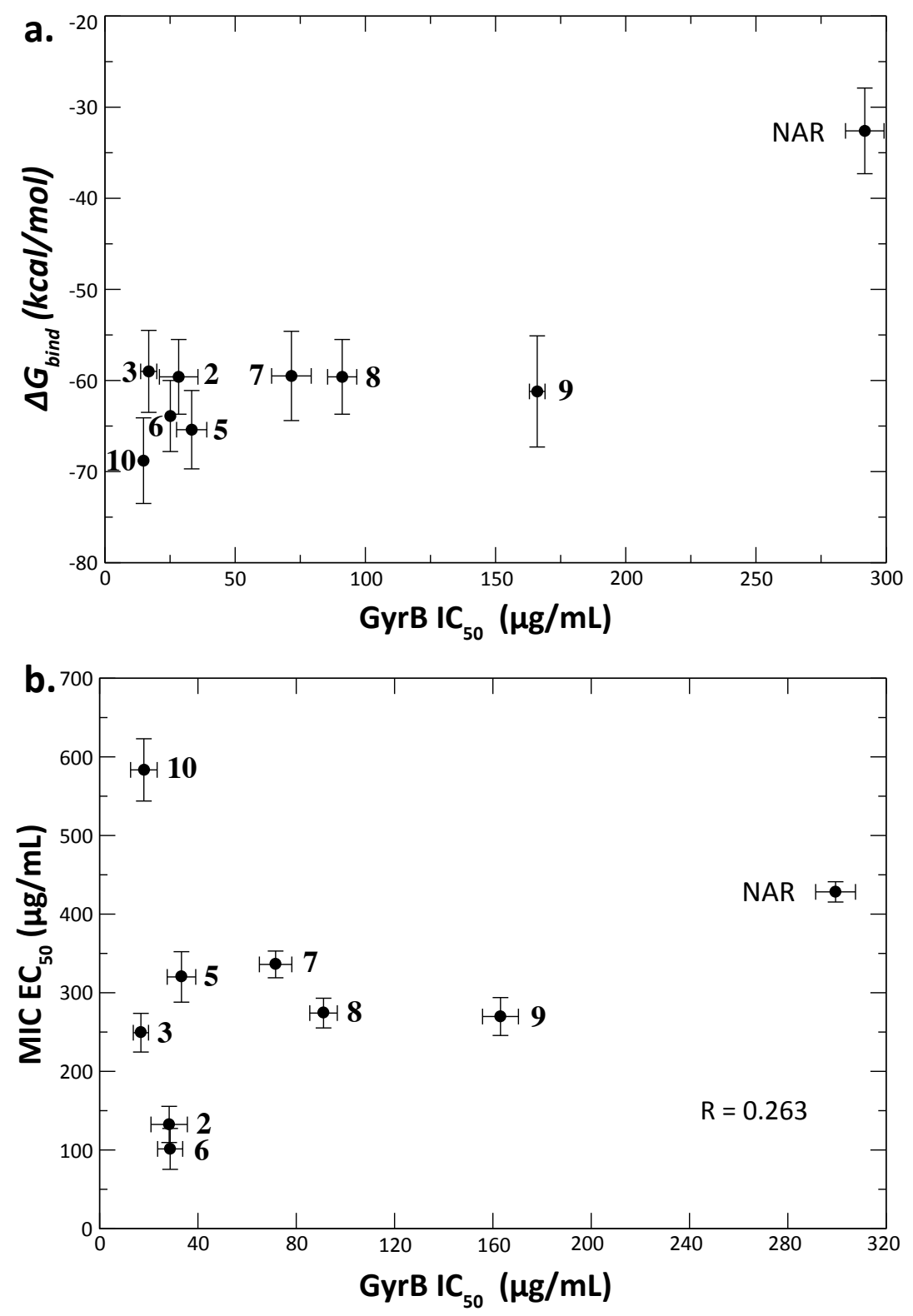

Figure 5. Measured DNA gyrase inhibition correlates moderately well with predicted binding free energy but poorly with antimicrobial activity. Plotted in panel (a) are $E$. coli DNA gyrase $\mathrm{B}$ inhibitory concentrations $\left(\mathrm{GyrB} I \mathrm{IC}_{50}\right)$ and calculated approximate binding free energies. Although these values show a good linear correlation (Pearson $r=0.88$ ), the linear fit is dominated by naringinen, and the rank-order correlation is weak (Spearman rho $=0.27$ ). Plotted in panel (b) are correlations of DNA gyrase $B$ inhibition with growth inhibitory concentrations $\left(E_{50}\right)$ for naringenin (NAR) and all flavanone derivatives except 4.

\section{Conclusions}

Antimicrobial activity results from a combination of small-molecule uptake, targetbinding activity, intracellular degradation, and efflux. Of these, target-binding activity has been the most straightforward to optimize, and small-molecule uptake in Gram-negative 
bacteria has remained a challenging target. Recent advances in predicting uptake in such bacteria offer the promise to co-optimize candidate molecules for binding and uptake early in the lead generation and optimization process. Machine-learning-based approaches are powerful and computationally efficient in this regard but have thus far been most accurate in well sampled areas of chemical space. Simulation-based prediction offers a more computationally intensive but more flexible approach to optimizing Gram-negative bacterial uptake. Here, we demonstrate that molecular simulations can be used in a prospective manner to select and order derivatives for synthesis and characterization. This simulation-guided engineering was performed for a target and scaffold that the simulation and analysis methodology had not been previously optimized or parameterized for, thus constituting a strong predictive test. This method can be combined with high-throughput prediction of target binding free energies to further enhance computational lead optimization and in the future also coupled to predictions of efflux. We note that compound D1 (Table S2), with a greatly improved predicted binding $\Delta \mathrm{G}$ of $-80 \mathrm{kcal} / \mathrm{mol}$ and moderate permeability of $3.5 \times 10^{-6} \mathrm{~cm} / \mathrm{s}$, would form a good starting point for such a joint optimization. At least for flavonoid DNA gyrase inhibitors, the molecules used were uptake-dominated in that changes to bacterial membrane permeability capture $86 \%$ of the variation in antimicrobial activity. This approach for lead optimization can be readily automated, and we provide code for the requisite analyses. We note that efflux can also be a major determinant to intracellular compound accumulation and antimicrobial activity depending on the compound chemistry. The relative effects of membrane permeability and efflux will vary on the compound set, so our approach should also be evaluated on other compound chemistries to determine generalizability. It nonetheless serves as a proof of concept for increasing antimicrobial activity by optimizing compound uptake.

\section{Methods}

Compound synthesis. Synthesis of hydrazone derivatives is described in brief below; materials, NMR spectra, yields, and peak assignments are given in the Supplement. 1.5 equiv. of the hydrazine $\left(\mathrm{R}-\mathrm{NH}-\mathrm{NH}_{2}\right)$ substituent was added to 1 equiv. of naringenin (1) dissolved in ethanol containing 0.01 equiv. of $10 \%$ acetic acid in ethanol. The mixture was kept stirring and heated until reflux under argon atmosphere until disappearance of the starting compound. The solvent was evaporated, and the residue was extracted with ethyl acetate, dried with anhydrous sodium sulfate, and further purified by preparative thin-layer chromatography (TLC, $n$-hexane:acetone, 1:1) to obtain the corresponding hydrazone (Figures S3-S8). Compound $\mathbf{4}$ had poor synthetic yield, poor OMV uptake, and high $\mathrm{MIC} \mathrm{EC}_{50}$ value, so DNA gyrase $\mathrm{B}$ inhibition was not determined.

Docking calculations. The crystallographic structure of DNA Gyrase B ATP binding domain of Escherichia coli in complex with a small molecule inhibitor (PDB ID: 4KFG; Figure $1 \mathrm{~A})^{37}$, was used in all molecular docking studies. The docking software was selected by evaluating its ability to reproduce the crystallographic pose of the bound inhibitor as follows. Both the Molecular Operating Environment (MOE) v2019.1 ${ }^{38}$ and Autodock Vina 1.1.2 ${ }^{39}$ packages were able to reproduce the pose of the co-crystallized 
ligand, although key predicted interactions differed. Both docking protocols reproduced interactions between the methylamino moiety in ring $A$ and Asp73 and also preserving the hydrogen-bond network connecting the pyrimidine ring with Asp73, Arg76 and Thr165 (Figure 1A), but the orientation of the fused octahydropyrrole substituent and its interaction with Asn46 (Figure S2) was better reproduced with MOE than with Vina. Based on these criteria, MOE was chosen for assessment of derivative binding poses.

Sixty-one candidate derivative molecules were built in MOE and minimized using the Amber10:ETH force field. Docking was performed using the Dock module of MOE with default options, using the co-crystallized inhibitor to define the active site. The docking algorithm used was Triangle Matcher with the London dG scoring function (initial docking placement and scoring methodologies, 30 poses) followed by a rigid receptor with a GBVI/WSA dG (post-placement refinement and scoring methodology, 5 poses). The candidate derivatives that maintained the correct orientation inside the ATPase binding pocket and kept key drug-protein interactions observed in the co-crystallized inhibitor were further evaluated for approximate binding free-energies and outer membrane permeabilities.

Small molecule parameterization. Simulations for binding free-energies were performed using the CHARMM $36^{40}$ and CGenFF force fields ${ }^{41}$, and those for permeability were performed using GROMOS96. Small-molecule topologies for CHARMM36 force field were obtained using the CGenFF server ${ }^{41}$ and converted to GROMACS format using the cgenff_charmm2gmx.py script. For permeability calculations, as in previous work ${ }^{12}$, GROMOS96 parameters for all compounds were assigned using the PRODRG server ${ }^{42}$ with partial charges assigned using the AM1-BCC charge model ${ }^{43}$ in Antechamber $1.27^{44}$.

Approximate binding free-energy calculations. Approximate relative free energies of binding were estimated using molecular mechanics-Poisson Boltzmann surface area $(\mathrm{MM}-\mathrm{PBSA})^{45}$ calculations based on molecular dynamics (MD) simulations of the liganddrug complex in GROMACS ${ }^{46}$ using the CHARMM36 and CGenFF force as follows. The 29 compounds passing docking evaluation were simulated via MD starting from their top-ranked binding pose. The system was solvated with TIP3P waters and counter ions was added sufficient to obtain charge neutralization. The system was energy-minimized and then equilibrated for 10 ps under NVT and 1 ns under NPT conditions (Nosé-Hoover thermostat, Parrinello-Rahman barostat), keeping all heavy atoms position-restrained. After equilibration, a 50-ns unrestrained NPT run followed, from which the last 30 ns were considered production. Long-range electrostatics were treated via Particle Mesh Ewald $(\mathrm{PME})^{47}$, with 1.2-nm short-range cut-offs for electrostatic and van der Waals interactions $(1.2 \mathrm{~nm})$. SETTLE and LINCS ${ }^{48}$ algorithms were used to constrain all bond lengths. Approximate relative free-energies of binding (total and per residue contributions) were calculated with g_mmpbsa ${ }^{49}$ for the last $30 \mathrm{~ns}$ of the simulation run.

Prediction of outer-membrane permeability. Predictions of outer-membrane permeability were performed using parameters previously published ${ }^{12}$. Briefly, OmpF was used as a model outer membrane porin and was simulated in a bacterial outer membrane patch 
consisting of 120 LPS in the outer leaflet and 276 PVPE:68 PVPG:37PVPV-DPG in the inner leaflet. Simulations were performed in $\mathrm{GROMACS}^{46}$ using explicit water. Each small molecule compound was first slowly pulled through the porin, and then this pulling simulation was used to seed umbrella sampling and permeability calculations. The single molecule was placed in bulk water at roughly $\sim 1.2 \mathrm{~nm}$ from the first OmpF monomer and all overlapping waters removed. The molecule was then pulled to the opposite side of the membrane over $100 \mathrm{~ns}$, using a spring constant of $1000 \mathrm{~kJ} \mathrm{~mol}^{-1} \mathrm{~nm}^{-2}$ and a pull rate of $0.085 \pm 0.005 \mathrm{~nm} \mathrm{~ns}^{-1}$ to obtain an initial path from which starting points for umbrella sampling were chosen at even z-intervals. Twenty-six (26) umbrella windows, with a mean width of $0.35 \pm 0.02 \mathrm{~nm}$ were used to sample the $z$ coordinate path and $40 \mathrm{~ns}$ of molecular simulation was performed per window ( $>1 \mu$ s total sampling per molecule).

The inhomogeneous solubility-diffusion (ISD) model was used to estimate drug permeabilities as in previous work $^{12}$ using the following equation:

$$
\frac{1}{P_{d r u g}}=R_{d r u g}^{p}=\int_{z_{1}}^{z_{2}} \frac{\exp [\Delta G(z)]}{D(z)} d z
$$

where $P_{\text {drug }}$ is permeability, $R^{p}$ is resistance to permeation, $\beta=1 / k_{B} T, \Delta G(z)$ is the potential of mean force from umbrella sampling, $D(z)$ is the local diffusivity coefficient, also estimated from umbrella sampling, and $z$ is the relative position of the molecule along the pore axis. Error estimation for the PMF curves were estimated by bootstrapping over umbrella sampling windows, sampled at intervals equal to the calculated autocorrelation times $\left(\tau_{z}\right)$ in each window. PMF curves are plotted in Fig. S9. We also performed validation studies where we rotated compounds 180 degrees at the beginning of the pulling run and used that for umbrella sampling and subsequent PMF and permeability calculation. These validation data yield permeabilities within $\sim 1.1$ orders of magnitude, adding an uncertainty term but preserving the log-linear relationship between predicted permeability and measured compound uptake.

Outer-membrane vesicle swelling assays. Assays were performed as in prior work ${ }^{12}$. Briefly, $5 \mu \mathrm{L}$ of $E$. coli MG1655, $\Delta \mathrm{OmpC}$ (kind gift of Linus Sandegren) was inoculated in $5 \mathrm{~mL}$ LB broth and incubated overnight at $37^{\circ} \mathrm{C} / 200 \mathrm{rpm}$. This culture was then seeded into $250 \mathrm{~mL}$ of fresh LB broth and incubated for $4 \mathrm{~h}$ at $37^{\circ} \mathrm{C} / 100 \mathrm{rpm}$ or to $\mathrm{OD}_{600}$ between 1.2 and 1.4. This culture was centrifuged for $20 \mathrm{~min}$ at $10,000 \mathrm{x} \mathrm{g}$ and $4{ }^{\circ} \mathrm{C}$. The supernatant was then sterile-filtered through a $0.22 \mu \mathrm{m}$ filter followed by ultracentrifugation for $4 \mathrm{~h}$ at 150,000x $\mathrm{g}, 4^{\circ} \mathrm{C}$. The supernatant was immediately decanted, and the pellet was resuspended in $1 \mathrm{~mL}$ MES buffer $\mathrm{pH}$ 6.0, for DLS measurements. Size measurements, cryo-electron micrographs, and SDS-PAGE of the OMV preparations are also given in our prior work ${ }^{12}$.

Each compound was dissolved in $100 \mu \mathrm{L}$ of MES buffer $\mathrm{pH} 6.0$ to a final concentration of $9 \mathrm{mM}^{12,50,51}$. To measure vesicle swelling, $6 \mu \mathrm{L}$ of the OMV suspension was added to $6 \mu \mathrm{L}$ MES buffer containing $2 \mu \mathrm{L}$ MES (control) or $2 \mu \mathrm{L}$ of the desired molecule, to a total volume of $14 \mu \mathrm{L}$. The light-scattering autocorrelation function was measured over 60 seconds using a $660 \mathrm{~nm}$ laser on an AvidNano W130i instrument (Avid Nano, High Wycombe, UK). All measurements were performed using a quartz cuvette 
and processed with pUnk 1.0.0.3 (Avid Nano, High Wycombe, UK). At least 3 biological replicates of 4 technical replicates each were performed per compound tested. No unexpected or unusually high safety hazards were encountered.

DNA gyrase inhibition assays. DNA gyrase supercoiling activity was assessed by measuring the conversion of relaxed plasmid pBR322 DNA to the supercoiled form ${ }^{52,53}$. Inhibition assays were carried out using an $E$. coli gyrase supercoiling assay kit from Inspiralis (Norwich Research Park, Colney Lane, Norwich. UK) according to manufacturer instructions. Briefly, in $30 \mu \mathrm{L}$ reaction mixtures containing the DNA gyrase assay buffer [35 mM Tris- $\mathrm{HCl}$ (pH 7.5), $24 \mathrm{mM} \mathrm{KCl,} 4 \mathrm{mM} \mathrm{MgCl}$, 2 mM DTT, $1.8 \mathrm{mM}$ spermidine, 1 $\mathrm{mM}$ ATP, $6.5 \%(\mathrm{w} / \mathrm{v})$ glycerol and $0.1 \mathrm{mg}$ of bovine serum albumin (BSA)/mL], $150 \mathrm{ng}$ of relaxed pBR322, $2-3 \mu \mathrm{L}$ of purified DNA gyrase and $3 \mu \mathrm{L}$ of serial test compounds in a serial 2-fold dilution. $3 \mu \mathrm{L}$ DMSO was used as control. The mixture was incubated for 30 min at $37{ }^{\circ} \mathrm{C}$, after which the reaction was stopped by adding $30 \mu \mathrm{L}$ of STEB [40\% (w/v) sucrose, $100 \mathrm{mM}$ Tris- $\mathrm{HCl}$ ( $\mathrm{pH}$ 8), $10 \mathrm{mM}$ EDTA, $0.5 \mathrm{mg} / \mathrm{mL}$ bromophenol blue] and 30 $\mu \mathrm{L}$ of chloroform/isoamyl alcohol (v:v, 24:1), vortexed briefly and centrifuged for 1 minute. Then, $20 \mu \mathrm{L}$ of the aqueous upper blue phase was loaded onto a $1 \%(\mathrm{w} / \mathrm{v})$ agarose gel in TAE buffer (Tris/EDTA, pH 8.0) and an electrophoresis followed during $2 \mathrm{~h}$ at $85 \mathrm{~V}$. The gel was then stained with SYBR ${ }^{\text {TM }}$ Safe DNA Gel Stain in water (15 min), destained (5$10 \mathrm{~min}$ ) and visualized under a trans-illuminator. The inhibitory effect of flavonoid on DNA gyrase was assessed by determining the concentration of flavonoid required to inhibit $50 \%$ of the supercoiling activity of the enzyme $\left(\mathrm{IC}_{50}\right)$. All determinations were performed as least in triplicate. A total of 1 unit $(U)$ of enzyme activity was defined as the amount of DNA gyrase that converted $150 \mathrm{ng}$ of relaxed pBR322 DNA to the supercoiled form in 30 min.

Antimicrobial activity. Antibacterial activity was assessed as the concentration that inhibits the growth of $50 \%$ of $E$. coli MG1655 strain in broth culture (MIC EC $\mathrm{E}_{50}$ ). The $\mathrm{EC}_{50}$ of the test compounds was determined by microtiter broth dilution performed in 96-well microplates as described by Wiegand et al ${ }^{54}$. Bacterial cultures were prepared by inoculating $5 \mathrm{~mL}$ of Mueller Hinton broth with $5 \mu \mathrm{L}$ of a bacterial glycerol stock followed by incubation at $37^{\circ} \mathrm{C} / 200 \mathrm{rpm}$ for $5 \mathrm{~h}$. The turbidity of the culture was adjusted to $\mathrm{A}_{600}$ 0.07 to 0.1 ( $0.5 \mathrm{McFarland}$ ) by dilution with fresh medium. Each compound was tested in twofold dilutions, using ciprofloxacin and novobiocin as internal controls on each plate. $5 \mu \mathrm{L}$ of bacterial culture was added to $100 \mu \mathrm{L}$ of compound diluted in Mueller Hinton broth in each well. After incubating the plates for $12-18 \mathrm{~h}$ at $37^{\circ} \mathrm{C}$, bacterial growth was examined by measuring the $A_{600}$ optical density (Multiskan FC, Thermo Fisher Scientific). All measurements were performed at least in triplicates. $\mathrm{EC}_{50}$ values were also obtained for highly potent and least potent compounds in a $\triangle T$ TolC E. coli strain (Keio JW5503 ${ }^{55}$ ); comparisons are given in Table S4.

\section{Acknowledgements}


This work was supported by a Coulter Translational Partners award and a Wallenberg Academy Fellowship to P.M.K. The authors thank A. Villamil Giraldo for helpful discussions, M-U. Ferreira for the gift of compounds, D. dos Santos for software, and L. Sandegren for the gift of bacterial strains. Compute time was provided by the HPC2N center supported by the Swedish National Infrastructure for Computing.

\section{References}

1. Review on Antimicrobial Resistance a. Tackling drug-resistant infections globally : final report and recommendations / the Review on Antimicrobial Resistance chaired by Jim O'Neill.). Review on Antimicrobial Resistance (2016).

2. Spellberg B, et al. The epidemic of antibiotic-resistant infections: a call to action for the medical community from the Infectious Diseases Society of America. Clin Infect Dis 46, 155-164 (2008).

3. Boucher HW, et al. Bad bugs, no drugs: no ESKAPE! An update from the Infectious Diseases Society of America. Clin Infect Dis 48, 1-12 (2009).

4. Boucher HW, et al. 10 x '20 Progress--development of new drugs active against gramnegative bacilli: an update from the Infectious Diseases Society of America. Clin Infect Dis 56, 1685-1694 (2013).

5. Frantz S. Better antibiotics through chemistry. Nat Rev Drug Discov 3, 900-901 (2004).

6. Tacconelli E, et al. Discovery, research, and development of new antibiotics: the WHO priority list of antibiotic-resistant bacteria and tuberculosis. Lancet Infect Dis 18, 318327 (2018).

7. Silver LL. Challenges of antibacterial discovery. Clin Microbiol Rev 24, 71-109 (2011).

8. Winterhalter $\mathrm{M}$, Ceccarelli M. Physical methods to quantify small antibiotic molecules uptake into Gram-negative bacteria. Eur J Pharm Biopharm 95, 63-67 (2015).

9. Pages J-M, James CE, Winterhalter M. The porin and the permeating antibiotic: a selective diffusion barrier in Gram-negative bacteria. Nature Reviews Microbiology 6, 893 (2008).

10. Masi M, Refregiers M, Pos KM, Pages JM. Mechanisms of envelope permeability and antibiotic influx and efflux in Gram-negative bacteria. Nat Microbiol 2, 17001 (2017).

11. Vergalli J, et al. Porins and small-molecule translocation across the outer membrane of Gram-negative bacteria. Nat Rev Microbiol 18, 164-176 (2020). 
12. Ferreira RJ, Kasson P. Antibiotic uptake across gram-negative outer membranes: better predictions towards better antibiotics. ACS Infectious Diseases 5, 2096-2104 (2019).

13. Nikaido H. Molecular basis of bacterial outer membrane permeability revisited. Microbiol Mol Biol Rev 67, 593-656 (2003).

14. Richter MF, et al. Predictive compound accumulation rules yield a broad-spectrum antibiotic. Nature 545, 299-304 (2017).

15. Richter MF, Hergenrother PJ. The challenge of converting Gram-positive-only compounds into broad-spectrum antibiotics. Annals of the New York Academy of Sciences 1435, 18-38 (2019).

16. Acosta-Gutierrez S, et al. Getting Drugs into Gram-Negative Bacteria: Rational Rules for Permeation through General Porins. ACS Infect Dis, (2018).

17. Bajaj $\mathrm{H}$, et al. Bacterial Outer Membrane Porins as Electrostatic Nanosieves: Exploring Transport Rules of Small Polar Molecules. ACS Nano 11, 5465-5473 (2017).

18. Prajapati JD, Kleinekathöfer U, Winterhalter M. How to Enter a Bacterium: Bacterial Porins and the Permeation of Antibiotics. Chem Rev, (2021).

19. Kutzner C, Grubmüller H, de Groot Bert L, Zachariae U. Computational Electrophysiology: The Molecular Dynamics of Ion Channel Permeation and Selectivity in Atomistic Detail. Biophysical Journal 101, 809-817 (2011).

20. Ziervogel BK, Roux B. The binding of antibiotics in OmpF porin. Structure 21, 76-87 (2013).

21. Bartsch A, et al. High-resolution experimental and computational electrophysiology reveals weak $\beta$-lactam binding events in the porin PorB. Scientific Reports 9, 1264 (2019).

22. Hajjar $E$, et al. Toward screening for antibiotics with enhanced permeation properties through bacterial porins. Biochemistry 49, 6928-6935 (2010).

23. Iyer R, Sylvester MA, Velez-Vega C, Tommasi R, Durand-Reville TF, Miller AA. Whole-CellBased Assay To Evaluate Structure Permeation Relationships for Carbapenem Passage through the Pseudomonas aeruginosa Porin OprD. ACS Infect Dis 3, 310-319 (2017).

24. Cama J, Henney AM, Winterhalter M. Breaching the Barrier: Quantifying Antibiotic Permeability across Gram-negative Bacterial Membranes. J Mol Biol 431, 3531-3546 (2019). 
25. Charifson PS, et al. Novel dual-targeting benzimidazole urea inhibitors of DNA gyrase and topoisomerase IV possessing potent antibacterial activity: intelligent design and evolution through the judicious use of structure-guided design and structure-activity relationships. J Med Chem 51, 5243-5263 (2008).

26. Sherer BA, et al. Pyrrolamide DNA gyrase inhibitors: optimization of antibacterial activity and efficacy. Bioorg Med Chem Lett 21, 7416-7420 (2011).

27. Tari LW, et al. Pyrrolopyrimidine inhibitors of DNA gyrase B (GyrB) and topoisomerase IV (ParE). Part I: Structure guided discovery and optimization of dual targeting agents with potent, broad-spectrum enzymatic activity. Bioorg Med Chem Lett 23, 1529-1536 (2013).

28. Trzoss $\mathrm{M}$, et al. Pyrrolopyrimidine inhibitors of DNA gyrase B (GyrB) and topoisomerase IV (ParE), Part II: development of inhibitors with broad spectrum, Gram-negative antibacterial activity. Bioorg Med Chem Lett 23, 1537-1543 (2013).

29. Ehmann DE, Lahiri SD. Novel compounds targeting bacterial DNA topoisomerase/DNA gyrase. Curr Opin Pharmacol 18, 76-83 (2014).

30. Bisacchi GS, Manchester JI. A New-Class Antibacterial-Almost. Lessons in Drug Discovery and Development: A Critical Analysis of More than 50 Years of Effort toward ATPase Inhibitors of DNA Gyrase and Topoisomerase IV. ACS Infect Dis 1, 4-41 (2015).

31. Dorsey D. Determination that ALBAMYCIN (novobiocin sodium) capsule, 250 milligrams, was withdrawn from sale for reasons of safety or effectiveness. Federal-Register 76, 3143-3144 (2011).

32. Plaper A, Golob M, Hafner I, Oblak M, Solmajer T, Jerala R. Characterization of quercetin binding site on DNA gyrase. Biochem Biophys Res Commun 306, 530-536 (2003).

33. Wu T, Zang X, He M, Pan S, Xu X. Structure-activity relationship of flavonoids on their anti-Escherichia coli activity and inhibition of DNA gyrase. J Agric Food Chem 61, 81858190 (2013).

34. Fang $Y$, et al. 3D-QSAR and docking studies of flavonoids as potent Escherichia coli inhibitors. Sci Rep 6, 23634 (2016).

35. Ferreira RJ, et al. Optimizing the flavanone core toward new selective nitrogencontaining modulators of ABC transporters. Future Med Chem 10, 725-741 (2018).

36. De Gier J, Mandersloot JG, Van Deenen LLM. Lipid composition and permeability of liposomes. Biochimica et Biophysica Acta (BBA) - Biomembranes 150, 666-675 (1968). 
37. Tari LW, et al. Tricyclic GyrB/ParE (TriBE) inhibitors: a new class of broad-spectrum dualtargeting antibacterial agents. PLoS One 8, e84409 (2013).

38. Vilar S, Cozza G, Moro S. Medicinal chemistry and the molecular operating environment (MOE): application of QSAR and molecular docking to drug discovery. Curr Top Med Chem 8, 1555-1572 (2008).

39. Trott O, Olson AJ. AutoDock Vina: improving the speed and accuracy of docking with a new scoring function, efficient optimization, and multithreading. J Comput Chem 31, 455-461 (2010).

40. Huang J, MacKerell AD, Jr. CHARMM36 all-atom additive protein force field: validation based on comparison to NMR data. J Comput Chem 34, 2135-2145 (2013).

41. Vanommeslaeghe $K$, MacKerell AD, Jr. Automation of the CHARMM General Force Field (CGenFF) I: bond perception and atom typing. J Chem Inf Model 52, 3144-3154 (2012).

42. Schuttelkopf AW, van Aalten DM. PRODRG: a tool for high-throughput crystallography of protein-ligand complexes. Acta Crystallogr D Biol Crystallogr 60, 1355-1363 (2004).

43. Jakalian A, Jack DB, Bayly $\mathrm{Cl}$. Fast, efficient generation of high-quality atomic charges. AM1-BCC model: II. Parameterization and validation. J Comput Chem 23, 1623-1641 (2002).

44. Wang J, Wang W, Kollman PA, Case DA. Automatic atom type and bond type perception in molecular mechanical calculations. J Mol Graph Model 25, 247-260 (2006).

45. Kollman PA, et al. Calculating structures and free energies of complex molecules: combining molecular mechanics and continuum models. Acc Chem Res 33, 889-897 (2000).

46. Pronk S, et al. GROMACS 4.5: a high-throughput and highly parallel open source molecular simulation toolkit. Bioinformatics 29, 845-854 (2013).

47. Darden T, York D, Pedersen L. Particle Mesh Ewald - an N.Log(N) Method for Ewald Sums in Large Systems. Journal of Chemical Physics 98, 10089-10092 (1993).

48. Hess B. P-LINCS: a parallel linear constraint solver for molecular simulation. J Chem Theory Comput 4, 116-122 (2008).

49. Kumari R, Kumar R, Open Source Drug Discovery C, Lynn A. g_mmpbsa--a GROMACS tool for high-throughput MM-PBSA calculations. J Chem Inf Model 54, 1951-1962 (2014). 
50. Nikaido $\mathrm{H}$, Rosenberg EY. Porin channels in Escherichia coli: studies with liposomes reconstituted from purified proteins. J Bacteriol 153, 241-252 (1983).

51. Yoshimura F, Nikaido H. Diffusion of beta-lactam antibiotics through the porin channels of Escherichia coli K-12. Antimicrob Agents Chemother 27, 84-92 (1985).

52. Hallett P, Grimshaw AJ, Wigley DB, Maxwell A. Cloning of the DNA gyrase genes under tac promoter control: overproduction of the gyrase A and B proteins. Gene $93,139-142$ (1990).

53. Ali JA, Jackson AP, Howells AJ, Maxwell A. The 43-kilodalton N-terminal fragment of the DNA gyrase B protein hydrolyzes ATP and binds coumarin drugs. Biochemistry 32, 27172724 (1993).

54. Wiegand I, Hilpert K, Hancock RE. Agar and broth dilution methods to determine the minimal inhibitory concentration (MIC) of antimicrobial substances. Nature protocols $\mathbf{3}$, 163-175 (2008).

55. Baba T, et al. Construction of Escherichia coli K-12 in-frame, single-gene knockout mutants: the Keio collection. Mol Syst Biol 2, 20060008 (2006). 